\title{
Ultrasensitive picomolar detection of aqueous acids in microscale fluorescent droplets
}

Hui Wanga, Zixiang Wei ${ }^{b}$, Sergei I. Vaginc, Xuehua Zhang ${ }^{b}$, Bernhard Rieger ${ }^{c}$, Alkiviathes Meldruma, $^{a}{ }^{*}$

\author{
a Department of Physics, University of Alberta, 11335 Saskatchewan Dr NW, Edmonton, Alberta T6G \\ 2M9, Canada \\ ${ }^{\mathrm{b}}$ Department of Chemical and Materials Engineering, University of Alberta, Edmonton, Alberta T6G \\ $1 \mathrm{H} 9$, Canada \\ c Department of Chemistry, Technical University of Munich, Lichtenbergstraße 4, 85747 Garching bei \\ München, Germany
}

\section{Supporting Information}

\section{Errors and uncertainties}

The errors and uncertainties from all figures represent the standard deviation of the sensing signals from four droplets with similar sizes. We chose four fluorescent octanol droplets with similar sizes in the fluorescence microscope image. The microscope image was split into the red, green, and blue channels by using Image J software. Then we chose one or a group of 100 pixels on the center of one octanol droplet to get the pixel intensity in red, green, and blue channel. The signal $S$ will be defined as $R /(R+G+B)$. The error bars in the graphs represent the standard deviation of four independent measurements on different droplets. In the concentration measurements (i.e., Fig. 5) the errors represent the standard deviation from four measurements of the concentration (obtained by measuring $S$ and concerting to $C_{o}$ via the calibration curves). The error in the partition coefficients were found by calculating $P_{o / w}$ four times from the four concentration measurements) and finding the standard deviation. We further note that the calibration curves are based on equations that were selected to fit closely to the known data points in order to extract concentrations at signal levels between the calibration values. Because the protonation goes through at least two equilibria and is likely accompanied by associate formation, the chemical system was judged too complicated to permit a physically-derived model that could completely describe the system. The error (and thus the LoD also) could be decreased by averaging over a greater number of image pixels from the droplet images. To be somewhat conservative, we tried initially taking only a single pixel near the center of the droplet, but if instead we average over 100 pixels the error decreased by approximately one order of magnitude.

\section{Repeating the LoD calculation}

The LoD for acid sensing when using a higher np-P4VB concentration ( $1.46 \mathrm{mM}$ ) can be estimated 
in the same way as described in the main text. Here, however, we took the lowest measured concentration at $C_{w}=10^{-8} \mathrm{M}$ in order to extrapolate the LoD. The sensitivity $S$ was estimated from the difference in the sensing signals between $10^{-8} \mathrm{M}$ salicylic acid and the blank, both after 25 minutes. The calculated LOD was $1.5 \times 10^{-9} \mathrm{M}$, which is somewhat worse than that from lower np-P4VB concentration.

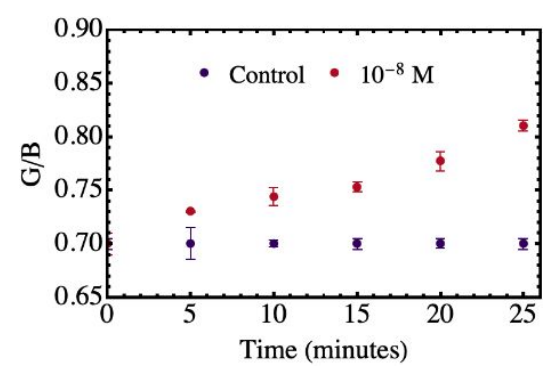

Fig. S1. The relationship between the sensing signal $(G / B)$ and time for a sample of $C_{P 4 V B}=1.46 \mathrm{mM}$ with $\mathrm{Cw}=10 \mathrm{nM}$ and a blank. The diameter of the droplets was about $15 \mu \mathrm{m}$.

Table S1. Model fitting parameters for droplets of various diameters, for two concentrations of salicylic acid in water $\left(\mathrm{C}_{\mathrm{w}}\right)$.

\begin{tabular}{|c|c|c|c|c|}
\hline$\left(C_{w}=10^{-}\right.$ & $\begin{array}{l}\text { Scaling } \\
\text { B } \\
\text { M) }\end{array}$ & $\begin{array}{l}K \\
(\mathrm{~m} / \mathrm{s})\end{array}$ & $t_{0}(s)$ & $D\left(m^{2} / s\right)$ \\
\hline 4.1 um & 0.192 & $6.15 \times 10^{-7}$ & 377 & $3.24 \times 10^{-5}$ \\
\hline $5.6 \mathrm{um}$ & 0.193 & $5.51 \times 10^{-7}$ & 464 & $1.02 \times 10^{-5}$ \\
\hline $7.2 \mathrm{um}$ & 0.192 & $7.91 \times 10^{-7}$ & 587 & $1.07 \times 10^{-5}$ \\
\hline 8.4 um & 0.193 & $7.41 \times 10^{-7}$ & 683 & $1.03 \times 10^{-5}$ \\
\hline $13.1 \mathrm{um}$ & 0.198 & $9.50 \times 10^{-7}$ & 763 & $1.04 \times 10^{-5}$ \\
\hline \multicolumn{5}{|c|}{$C_{w}=10^{-6} \mathrm{M}$} \\
\hline $5.3 \mathrm{um}$ & 0.090 & $6.97 \times 10^{-7}$ & 341 & $1.26 \times 10^{-5}$ \\
\hline 6.3 um & 0.091 & $7.47 \times 10^{-7}$ & 422 & $1.59 \times 10^{-5}$ \\
\hline 7.2 um & 0.091 & $6.94 \times 10^{-7}$ & 529 & $1.15 \times 10^{-5}$ \\
\hline 9.4 um & 0.091 & $7.62 \times 10^{-7}$ & 621 & $1.19 \times 10^{-5}$ \\
\hline
\end{tabular}




\section{Droplet size distribution}

The droplet size distribution was obtained by manually measuring the droplet diameters with ImageJ and calibrating the pixel counts using the image scale bars. The resulting size distribution with a lognormal fit is shown in Fig. S2. The mean is 10.0 micrometers with a standard deviation of 3.8 micrometers.

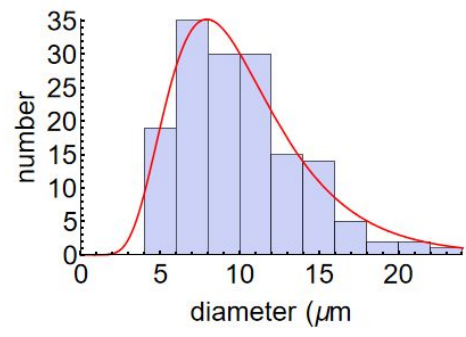

Fig. S2. The histogram of the size distribution obtained from 150 droplets. 